International Journal of Applied Mathematics

Volume 33 No. $4 \quad 2020,585-590$

ISSN: 1311-1728 (printed version); ISSN: 1314-8060 (on-line version)

doi: http://dx.doi.org/10.12732/ijam.v33i4.3

\title{
COMPARISON ON THE ROBUSTNESS AGAINST ERASURE RATES OF NUMERICALLY ERASURE-ROBUST FRAMES
}

\author{
Yang Liu \\ Shenzhen Technology University \\ Shenzhen, Guangdong 518118, CHINA
}

\begin{abstract}
In this paper, we mainly study the subframe condition numbers of numerically erasure-robust frames, which can be used in signal processing, and establish a comparison theorem on the condition numbers of different frames, which can be applied to inverse problems for signal processing.
\end{abstract}

AMS Subject Classification: 65F15, 15A18, 65Y99

Key Words: singular values; conditional numbers; frames

\section{Introduction}

The numerically erasure-robust frames (NERF) has been used to give a precise characterization of erasure robustness in information transmission.

Gribonval and Nielsen in [3] studied sparse representations of signals from a general dictionary in a Banach space $X$, that is is a family of unit vectors $\left\{f_{k}\right\}_{k \in \mathcal{K}}$ with dense span in $X$ ( $\mathcal{K}$ is a finite or countable index set) and gave sufficient conditions for having a unique sparse representation of a signal from the dictionary with respect to a large class of admissible sparseness measures. In [1], Fickus and many others used ideas from the theory of group to construct erasure-robust frames. For frames with diemnsions increasing to infinity, thee has been some relevant work such as [4], [8], [5], [7], and [6], etc.

In [2], Fickus and Mixon asked an open question whether a $(p, C)$-NERF is also a $(p \prime, C)$-NERF for every $p^{\prime} \in[0, p)$. In this article, we study the relations between the numerically erasure-robustness of a frame and its subframes generally and answer the open question particularly.

Received: January 17, 2020

(c) 2020 Academic Publications 


\section{On the robustness against $p$-erasure and $p^{\prime}$-erasure of a frame}

We first establish a lemma and give some examples on whether a $(p, C)$-NERF is a $(p /, C)$-NERF for $p^{\prime} \in[0, p)$.

Lemma 1. For any $M$ by $N$ frame $\left\{f_{1}, f_{2}, \ldots, f_{N}\right\}$ of a Hilbert space, let $\mathcal{S}:=\{1,2, \ldots, N\}$, then

$$
\max _{\mathcal{K} \subseteq \mathcal{S},|\mathcal{K}|=(1-p) N} \operatorname{Cond}\left(F_{\mathcal{K}}\right) \geq \max _{\mathcal{K}^{\prime} \subseteq \mathcal{S},\left|\mathcal{K}^{\prime}\right|=\left(1-p^{\prime}\right) N} \operatorname{Cond}\left(F_{\mathcal{K}^{\prime}}\right)
$$

for any $0 \leq p \leq 1$ and $0 \leq p^{\prime}<p$. In particular and in other words, there always exists some subframe, which survives the deletion of some column vectors in a frame, not as well-conditioned as the original frame.

Proof. By the spectral theorem,

$$
\lambda_{\max }\left(\sum_{\mathcal{K} \subseteq \mathcal{K}^{\prime},|\mathcal{K}|=(1-p) N} F_{\mathcal{K}} F_{\mathcal{K}}^{*}\right)=\mathbf{v}^{*}\left(\sum_{\mathcal{K} \subseteq \mathcal{K}^{\prime},|\mathcal{K}|=(1-p) N} F_{\mathcal{K}} F_{\mathcal{K}}^{*}\right) \mathbf{v}
$$

for some $\mathbf{v}$ in the Hilbert space with $\|\mathbf{v}\|=1$ and

$$
\lambda_{\min }\left(\sum_{\mathcal{K} \subseteq \mathcal{K}^{\prime},|\mathcal{K}|=(1-p) N} F_{\mathcal{K}} F_{\mathcal{K}}^{*}\right)=\mathbf{w}^{*}\left(\sum_{\mathcal{K} \subseteq \mathcal{K}^{\prime},|\mathcal{K}|=(1-p) N} F_{\mathcal{K}} F_{\mathcal{K}}^{*}\right) \mathbf{w}
$$

for some $\mathbf{w}$ in the Hilbert space with $\|\mathbf{w}\|=1$. Moreover,

$$
\max _{\mathcal{K} \subseteq \mathcal{K}^{\prime},|\mathcal{K}|=(1-p) N} \frac{\left(\mathbf{v}^{*} F_{\mathcal{K}} F_{\mathcal{K}}^{*} \mathbf{v}\right)^{\frac{1}{2}}}{\left(\mathbf{w}^{*} F_{\mathcal{K}} F_{\mathcal{K}}^{*} \mathbf{w}\right)^{\frac{1}{2}}} \geq \frac{\left(\mathbf{v}^{*}\left(\sum_{\mathcal{K} \subseteq \mathcal{K}^{\prime},|\mathcal{K}|=(1-p) N} F_{\mathcal{K}} F_{\mathcal{K}}^{*}\right) \mathbf{v}\right)^{\frac{1}{2}}}{\left(\mathbf{w}^{*}\left(\sum_{\mathcal{K} \subseteq \mathcal{K}^{\prime},|\mathcal{K}|=(1-p) N} F_{\mathcal{K}} F_{\mathcal{K}}^{*}\right) \mathbf{w}\right)^{\frac{1}{2}}} .
$$

Thus, it follows that

$$
\max _{\mathcal{K} \subseteq \mathcal{K}^{\prime},|\mathcal{K}|=(1-p) N} \frac{\left(\mathbf{v}^{*} F_{\mathcal{K}} F_{\mathcal{K}}^{*} \mathbf{v}\right)^{\frac{1}{2}}}{\left(\mathbf{w}^{*} F_{\mathcal{K}} F_{\mathcal{K}}^{*} \mathbf{w}\right)^{\frac{1}{2}}} \geq \frac{\lambda_{\max }^{\frac{1}{2}}\left(\sum_{\mathcal{K} \subseteq \mathcal{K}^{\prime},|\mathcal{K}|=(1-p) N} F_{\mathcal{K}} F_{\mathcal{K}}^{*}\right)}{\lambda_{\text {min }}^{\frac{1}{2}}\left(\sum_{\mathcal{K} \subseteq \mathcal{K}^{\prime},|\mathcal{K}|=(1-p) N} F_{\mathcal{K}} F_{\mathcal{K}}^{*}\right)} .
$$

On the other hand, for any fixed $\mathcal{K}^{\prime} \subseteq \mathcal{S}$ with $\left|\mathcal{K}^{\prime}\right|=\left(1-p^{\prime}\right) N$, we have

$$
F_{\mathcal{K}^{\prime}} F_{\mathcal{K}^{\prime}}^{*}=\frac{(N-p N-1) !\left(p N-p^{\prime} N\right) !}{\left(N-p^{\prime} N-1\right) !} \sum_{\mathcal{K} \subseteq \mathcal{K}^{\prime},|\mathcal{K}|=(1-p) N} F_{\mathcal{K}} F_{\mathcal{K}}^{*}
$$


and then it follows that

$$
\frac{\lambda_{\max }^{\frac{1}{2}}\left(\sum_{\mathcal{K} \subseteq \mathcal{K}^{\prime},|\mathcal{K}|=(1-p) N} F_{\mathcal{K}} F_{\mathcal{K}}^{*}\right)}{\lambda_{\min }^{\frac{1}{2}}\left(\sum_{\mathcal{K} \subseteq \mathcal{K}^{\prime},|\mathcal{K}|=(1-p) N} F_{\mathcal{K}} F_{\mathcal{K}}^{*}\right)}=\frac{\lambda_{\max }^{\frac{1}{2}}\left(F_{\mathcal{K}^{\prime}} F_{\mathcal{K}^{\prime}}^{*}\right)}{\lambda_{\min }^{\frac{1}{2}}\left(F_{\mathcal{K}^{\prime}} F_{\mathcal{K}^{\prime}}^{*}\right)}=\operatorname{Cond}\left(F_{\mathcal{K}^{\prime}}\right)
$$

Combine 5 and 7 , and it yields that there exist some $\mathbf{z}$ and $\mathbf{w}$ in the Hilbert space with $\|\mathbf{v}\|=1$ and $\|\mathbf{w}\|=1$, such that

$$
\max _{\mathcal{K} \subseteq \mathcal{K}^{\prime},|\mathcal{K}|=(1-p) N} \frac{\left(\mathbf{v}^{*} F_{\mathcal{K}} F_{\mathcal{K}}^{*} \mathbf{v}\right)^{\frac{1}{2}}}{\left(\mathbf{w}^{*} F_{\mathcal{K}} F_{\mathcal{K}}^{*} \mathbf{w}\right)^{\frac{1}{2}}} \geq \operatorname{Cond}\left(F_{\mathcal{K}^{\prime}}\right) .
$$

However, since

$$
\lambda_{\max }^{\frac{1}{2}}\left(F_{\mathcal{K}} F_{\mathcal{K}}^{*}\right) \geq\left(\mathbf{v}^{*} F_{\mathcal{K}} F_{\mathcal{K}}^{*} \mathbf{v}\right)^{\frac{1}{2}} \geq 0
$$

and

$$
\left(\mathbf{w}^{*} F_{\mathcal{K}} F_{\mathcal{K}}^{*} \mathbf{w}\right)^{\frac{1}{2}} \geq \lambda_{\min }^{\frac{1}{2}}\left(F_{\mathcal{K}} F_{\mathcal{K}}^{*}\right) \geq 0
$$

then

$$
\operatorname{Cond}\left(F_{\mathcal{K}}\right)=\frac{\lambda_{\text {max }}^{\frac{1}{2}}\left(F_{\mathcal{K}} F_{\mathcal{K}}^{*}\right)}{\lambda_{\text {min }}^{\frac{1}{2}}\left(F_{\mathcal{K}} F_{\mathcal{K}}^{*}\right)} \geq \frac{\left(\mathbf{v}^{*} F_{\mathcal{K}} F_{\mathcal{K}}^{*} \mathbf{v}\right)^{\frac{1}{2}}}{\left(\mathbf{w}^{*} F_{\mathcal{K}} F_{\mathcal{K}}^{*} \mathbf{w}\right)^{\frac{1}{2}}}
$$

Therefore, from 8 , it follows that

$$
\max _{\mathcal{K} \subseteq \mathcal{K}^{\prime},|\mathcal{K}|=(1-p) N} \text { Cond }\left(F_{\mathcal{K}}\right) \geq \operatorname{Cond}\left(F_{\mathcal{K}^{\prime}}\right) .
$$

Taking maximum on both sides, we have

$$
\begin{aligned}
\mathcal{K}^{\prime} \subseteq \mathcal{S},\left|\mathcal{K}^{\prime}\right|= & \left(1-p^{\prime}\right) N \\
& \max \left(\max _{\mathcal{K} \subseteq \mathcal{K}^{\prime},|\mathcal{K}|=(1-p) N} \operatorname{Cond}\left(F_{\mathcal{K}}\right)\right) \\
& \geq \max _{\mathcal{K}^{\prime} \subseteq \mathcal{S},\left|\mathcal{K}^{\prime}\right|=\left(1-p^{\prime}\right) N} \operatorname{Cond}\left(F_{\mathcal{K}^{\prime}}\right),
\end{aligned}
$$

but since

$$
\max _{\mathcal{K} \subseteq \mathcal{S},|\mathcal{K}|=(1-p) N} \operatorname{Cond}\left(F_{\mathcal{K}}\right) \geq \max _{\mathcal{K}^{\prime} \subseteq \mathcal{S},\left|\mathcal{K}^{\prime}\right|=\left(1-p^{\prime}\right) N}\left(\max _{\mathcal{K} \subseteq \mathcal{K}^{\prime},|\mathcal{K}|=(1-p) N} \operatorname{Cond}\left(F_{\mathcal{K}}\right)\right),
$$

then we obtain that

$$
\max _{\mathcal{K} \subseteq \mathcal{S},|\mathcal{K}|=(1-p) N} \operatorname{Cond}\left(F_{\mathcal{K}}\right) \geq \max _{\mathcal{K}^{\prime} \subseteq \mathcal{S},\left|\mathcal{K}^{\prime}\right|=\left(1-p^{\prime}\right) N} \operatorname{Cond}\left(F_{\mathcal{K}^{\prime}}\right),
$$

as desired. 
More generally, we have the following theorem for compact operators on Hilbert space:

Theorem 2. For any Hilbert space $\mathcal{H}$ and any compact operator $T$ on $\mathcal{H}$, one has

$$
\max _{\mathcal{V} \subseteq \mathcal{H}, \operatorname{dim}(\mathcal{V})=n} \operatorname{Cond}\left(\left.T\right|_{\mathcal{V}}\right) \geq \max _{\mathcal{V}^{\prime} \subseteq \mathcal{H}, \operatorname{dim}\left(\mathcal{V}^{\prime}\right)=n^{\prime}} \operatorname{Cond}\left(\left.T\right|_{\mathcal{V}^{\prime}}\right)
$$

and more strongly,

$$
\max _{\mathcal{V} \subseteq \mathcal{V}^{\prime}, \operatorname{dim}(\mathcal{V})=n} \operatorname{Cond}\left(\left.T\right|_{\mathcal{V}}\right) \geq \max _{\mathcal{V}^{\prime} \subseteq \mathcal{H}, \operatorname{dim}\left(\mathcal{V}^{\prime}\right)=n^{\prime}} \operatorname{Cond}\left(\left.T\right|_{\mathcal{V}^{\prime}}\right)
$$

for any $0 \leq n<n^{\prime}$, where $\left.T\right|_{\mathcal{V}}$ and $\left.T\right|_{\mathcal{V}^{\prime}}$ are the restrictions of $T$ to $\mathcal{V}$ and $\mathcal{V}^{\prime}$, respectively.

Proof. Use the fact that $T T^{*}$ and its restrictions to subspaces are all self-adjoint operators, and generalize the proof of the previous lemma.

For some frames, there may be some subframes that have condition numbers smaller than the condition number of the original frame. However, for any frame, there always exists some subframe whose condition number is greater than the condition number of the original frame. Here, we give some examples that a $(p, C)$-NERF is not necessarily a $(p /, C)$-NERF for some $p^{\prime} \in[0, p)$.

Example 3. Let

$$
F=\left[\begin{array}{ccc}
2 & 1 & 2 \\
1 & 1 & -1
\end{array}\right]
$$

$p=\frac{1}{3}$ and $p^{\prime}=0$. The condition number

$$
\operatorname{Cond}\left(F_{\mathcal{S}}\right)=\frac{6+\sqrt{10}}{\sqrt{26}} \approx 1.80
$$

where $\mathcal{S}=\{1,2,3\}$, and the condition number

$$
\operatorname{Cond}\left(F_{\mathcal{K}}\right)=\frac{1}{6}(7+\sqrt{13}) \approx 1.78
$$

for $\mathcal{K}=\{2,3\}$. However, the maximal condition number is

$$
\max _{\mathcal{K} \subseteq\{1,2,3\},|\mathcal{K}|=(1-p) N=\frac{2}{3} N} \operatorname{Cond}\left(F_{\mathcal{K}}\right)=\frac{1}{2}(7+3 \sqrt{5}) \approx 6.85 .
$$

Example 4. Let

$$
F=\left[\begin{array}{lll}
1 & 0 & 2 \\
0 & 1 & 0
\end{array}\right]
$$


$p=\frac{1}{3}$ and $p^{\prime}=0$. The condition number

$$
\text { Cond }\left(F_{\mathcal{S}}\right)=\sqrt{5}
$$

where $\mathcal{S}=\{1,2,3\}$, and the condition number

$$
\text { Cond }\left(F_{\mathcal{K}}\right)=1
$$

for $\mathcal{K}=\{1,2\}$. However, the maximal condition number is

$$
\max _{\mathcal{K} \subseteq\{1,2,3\},|\mathcal{K}|=(1-p) N=\frac{2}{3} N} \operatorname{Cond}\left(F_{\mathcal{K}}\right)=\infty .
$$

Example 5. Let

$$
F=\left[\begin{array}{lll}
0.4302 & 0.9049 & 0.4389 \\
0.1848 & 0.9797 & 0.1111
\end{array}\right]
$$

$p=\frac{1}{3}$ and $p^{\prime}=0$. The condition number

$$
\text { Cond }\left(F_{\mathcal{S}}\right)=5.0799
$$

where $\mathcal{S}=\{1,2,3\}$. The maximal condition number

$$
\begin{aligned}
& \max _{\mathcal{K} \subseteq\{1,2,3\},|\mathcal{K}|=(1-p) N=\frac{2}{3} N} \operatorname{Cond}\left(F_{\mathcal{K}}\right)=12.6571, \\
& \min _{\mathcal{K} \subseteq\{1,2,3\},|\mathcal{K}|=(1-p) N=\frac{2}{3} N} \operatorname{Cond}\left(F_{\mathcal{K}}\right)=5.8506,
\end{aligned}
$$

which means that all the subframes are not as well-conditioned as the original frame.

Remark 6. From this example, we can see that some frames can be retrenched in the number of spanning vectors, but may not be retrenched in condition number, because all the subframes of the fame in the example above have condition numbers greater than the original frame. However, obviously, the union of a tight frame with any set of vectors in the space can always be retrenched in condition number.

And we would like to make another remark:

Remark 7. The condition number of a $M$ by $N$ frame $F$ corresponds to the eccentricity of the at-most- $M$-dimensional ellipse $F^{T}\left(\mathbf{S}^{M-1}\right)$ in a $M$ dimensional subspace of $\mathbf{R}^{N}$ in geometry, and so the robustness of a NERF against erasures corresponds to the maximal eccentricity of the at-most- $M$ dimensional ellipses $F_{K}^{T}\left(\mathbf{S}^{M-1}\right)$ in a $M$-dimensional subspace, furthermore and more geometrically, the robustness of a NERF against erasures corresponds to the least conformality of the subframes of the NERF as maps. 


\section{Acknowledgment}

The author would like to thank Prof. Y. Wang for some helpful discussions. This work is partially supported by Shenzhen Municipal Finance for Research.

\section{References}

[1] M. Fickus, J. Jasper, D.G. Mixon, and J. Peterson, Grouptheoretic constructions of erasure-robust frames, Linear Algebra and its Applications, 479 (2015), 131-154.

[2] M. Fickus and D.G. Mixon, Numerically erasure-robust frames, Linear Algebra and its Applications, 437, No 6 (2012), 1394-1407.

[3] R. Gribonval and M. Nielsen, Highly sparse representations from dictionaries are unique and independent of the sparseness measure, Applied and Computational Harmonic Analysis, 22, No 3 (2007), 335-355.

[4] M.J. Lai and Y. Liu,The probabilistic estimates on the largest and smallest $q$-singular values of random matrices, Mathematics of Computation, 84, No 294 (2015), 1775-1794.

[5] Y. Liu, Probabilistic estimates of the largest strictly convex singular values of pregaussian random matrices, Journal of Mathematics and Statistics, 11, No 1 (2015), 7-15.

[6] Y. Liu and Y. Wang, On the decay of the smallest singular value of submatrices of rectangular matrices, Asian-European Journal of Mathematics, 9, No 4 (2016), \# 1650075.

[7] H. Ohlsson, A. Yang, R. Dong, and S. Sastry, Cprl-an extension of compressive sensing to the phase retrieval problem, In: Advances in Neural Information Processing Systems (2012), 1367-1375.

[8] M. Rudelson and R. Vershynin, Non-asymptotic theory of random matrices: Extreme singular values, In: Proc. of the International Congress of Mathematicians 2010 (ICM 2010), In 4 Volumes: Vol. I: Plenary Lectures and Ceremonies, Vols. II-IV: Invited Lectures, 1576-1602, World Scientific, 2010. 